\title{
Localized Parathyroid Gland Carcinoma
}

National Cancer Institute

\section{Source}

National Cancer Institute. Localized Parathyroid Gland Carcinoma. NCI Thesaurus. Code C7826.

A parathyroid gland carcinoma that has not spread to other anatomic sites. 